\title{
Complex Dynamical Behavior in the Shear-Displacement Model for Bulk Metallic Glasses during Plastic Deformation
}

\author{
Cun Chen, ${ }^{1,2}$ Shaokang Guan, ${ }^{2}$ and Liying Zhang $\mathbb{D}^{1}$ \\ ${ }^{1}$ School of Mathematics and Statistics, Zhengzhou University, 100 Science Road, Zhengzhou 450001, China \\ ${ }^{2}$ School of Materials Science and Engineering, Zhengzhou University, Zhengzhou 450001, China \\ Correspondence should be addressed to Liying Zhang; zhangly@zzu.edu.cn
}

Received 3 September 2018; Revised 17 November 2018; Accepted 19 November 2018; Published 3 December 2018

Academic Editor: Jose Manoel Balthazar

Copyright (c) 2018 Cun Chen et al. This is an open access article distributed under the Creative Commons Attribution License, which permits unrestricted use, distribution, and reproduction in any medium, provided the original work is properly cited.

\begin{abstract}
In this paper, a fresh shear-displacement model is developed for the plastic deformation of the bulk metallic glasses. The multiscale behavior in the shear banding process and the dynamics transition with the parameters are investigated in analytical form. We present a theoretical support for the transition from unstable states to stable states in the experiment by multiscale analysis and the stability analysis. With the small parameter increasing from negative to positive, the stability of the shear slipping displacement system changes, and there is a limit cycle at the transition stage. Meanwhile, the phase diagram and the power spectrum also suggest that there is dynamics transition with the parameter changing. Moreover, the complexity is analyzed for different disturbance parameters, and it is coincident with the fact that the solution is more irregular for larger disturbance. In addition, we find that the amplitude of solution decreases with the temperature decreasing, which is consistent with the experimental results that the amplitude of the serration is smaller and smaller as the temperature decreases.
\end{abstract}

\section{Introduction}

During the plastic deformation for the bulk metallic glasses (BMGs), the serrated flow manifests as a series of intermittent serration in the stress-strain (time) curve [1]. The system has complex dynamic behavior, which has not been revealed clearly by nonlinear theoretical analysis based on mathematical model. Each serration is considered to be associated with the formation of localized shear bands propagating along a certain shear plane, and thus the dynamics of serrations is closely related to the shear stability, the ductility of metallic glasses, or the temperature [2-7]. The experimental data analysis suggests that the shear avalanche can self-organize to a critical state in ductile metallic glasses [5] or can be in a chaotic state in metallic glasses with small plastic strain rate and the multifractal bursts at the transition stage [8]. Meanwhile, for the serrated flow signal, there exists temporal scaling behavior varying with different strain rates and temperatures [9], as well as the loading forces[10]. The selfsimilar scaling behavior only exists at certain temperature [9]. The temperature is an important factor influencing the microstructure of BMGs [11], which can further affect mechanical properties, such as the improvement of yield strength and plasticity $[12,13]$. Note that there are complex dynamical behaviors in the serrated flow based on the experimental data; therefore, we speculate that there exist various nonlinear dynamical behaviors based on the spatiotemporal dynamical model.

Although a few researches focused on the dynamics of the shear band during plastic deformation of metallic glasses [4, $5,14-17]$, the theoretical analysis based on a spatiotemporal dynamical model is quite needed to investigate the nonlinear phenomena in the plastic deformation. In 2014, it was noted that the serrated flow dynamics is influenced by the strain rates; we developed a dynamical model which involves the shear displacement (spatial information) and the argument time (temporal information) [18]. The model predicts the evolution of the shear displacement and the shear sliding speed in the shear band, all as a function of time. Considering the loading strain rate effects, we found various sizes of sliding events at different initial conditions. Although the model has constructed differential expressions to bridge the gap between the temporal evolution and the spatial interaction of shear bands, there is no further theoretical 


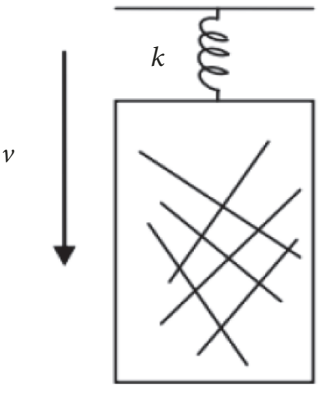

(a)

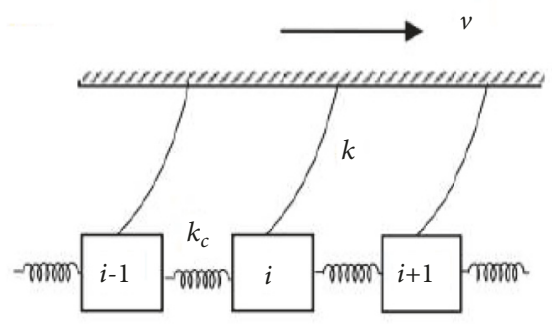

(b)
$k_{c}\left(U_{i}-U_{i-1}\right)+\sigma_{f}$

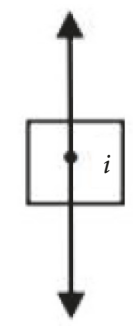

$k_{c}\left(U_{i+1}-U_{i}\right)+\sigma$

(c)

FIGURE 1: The schematic diagram of the plastic deformation considering the mutual interaction between the shear bands and force analysis for the multiple shear bands. (a) The multiple shear bands during the plastic deformation. (b) Chain of the blocks parallel to the direction of loading speed $v$. (c) The force diagram for the i-th block.

analysis for the serrated flow that has yet to be proposed, and the dynamical behavior transition with the parameters has not been illuminated in analytical form. Therefore, in the following work, we attempt to investigate how the dynamics transits with the parameters and the multiscale behavior in the shear banding process by applying the dynamical analysis and numerical simulation. In addition, we provide an explanation about how the temperature influences the solution of the dynamical system.

The aim of the present work is to explore the complex dynamical behavior in the shear-displacement model, as well as the influence of the temperature. The novel aspect of this work relates to two factors: (1) the multiscale analysis was conducted to evaluate the scaling behavior in the serrated plastic flow; (2) complex dynamical behavior can be deduced from this model at different conditions, which is corresponding to the chaos and self-organized behavior based on our previous experimental data analysis.

\section{The Sliding Shear-Displacement Model and Stability Analysis}

$\mathrm{Cu}_{50} \mathrm{Zr}_{45} \mathrm{Ti}_{5}$ metallic glasses were chosen as model materials. The tests were conducted at strain rates of $2.5 \times 10^{-2} \mathrm{~s}^{-1}$, $2.5 \times 10^{-3} s^{-1}, 2.5 \times 10^{-4} s^{-1}$, and $2.5 \times 10^{-5} s^{-1}$, respectively. The surface of the fractured sample exhibits abundant shear bands interlaced[1]. We introduce the springs connecting the neighboring blocks with different elastic coefficients, $k_{i}, i=$ $1,2, \ldots, N-1 ; N$ is the number of a chains of blocks that were coupled to each other; the mutual interaction between the shear bands is presented as $k_{i}\left(U_{i+1}-U_{i}\right)-k_{i-1}\left(U_{i}-U_{i-1}\right)$, where $U_{i}$ is the shear sliding displacement of the $i$ th block. Due to the limitation of the experiment method, so far the value of elastic coefficients of $k_{i}$ is hard to identify, and the formula $k_{i}\left(U_{i+1}-U_{i}\right)-k_{i-1}\left(U_{i}-U_{i-1}\right)$ is difficult to deal with in a partially differential equation. Therefore, theoretical analysis cannot be easily implemented. Using the average $k_{c}$ instead of $k_{i}$, we can also get convincing conclusion about the theory analysis and simulation results about the system of plastic deformation.

Therefore, without regard to the influences of the microstructures in glassy phase, we established a model considering mutual interaction between multiple shear bands [18], involving the shear displacement and the time; the sketch of the model is shown in Figure 1. The system contains a chain of blocks coupled to each other by harmonic springs with strength of $k_{c}$, and they were attached to the machine. The spring strength between the sample and the machine is $k$ (Figures $1(\mathrm{a})$ and $1(\mathrm{~b})$ ). The system is compressed at a loading speed, $v$ (Figure 1(a)). For the $i$ th block, the forces parallel to the loading direction are the internal stress, $\sigma U_{i}$, the interaction among the multiple shear bands, which is expressed as $U_{i+1}+U_{i-1}-2 U_{i}$, and the plastic shear resistance, $\sigma_{f}\left(\dot{U}_{i}\right)$, which is in the opposite direction of the loading direction (Figure 1(c)). Because the internal stress, $\sigma\left(U_{i}\right)$, can be written as $\sigma\left(U_{i}\right)=\sigma(0)-k U_{i}[4]$, the motion equation is given as follows:

$$
\begin{aligned}
\frac{4 M}{\pi d^{2}} \ddot{U}_{i}= & \sigma(0)-k U_{i}+k_{c}\left(U_{i+1}+U_{i-1}-2 U_{i}\right) \\
& -\sigma_{f}\left(\dot{U}_{i}\right)
\end{aligned}
$$

where $\sigma(0)$ is the initial internal stress, which is equal to the yield stress, $\sigma_{f 0}$, and $\sigma_{f}\left(\dot{U}_{i}\right)=\sigma_{f 0} /\left(1+A \dot{U}_{i}\right)$, which represents the shear resistance along the shear plane [5]. $d$ is the diameter of the sample. $M$ is the equivalent mass of the system, which consists of a metallic glass and a spring that represents the influence of the testing machine. $k=$ $E / L(1+S)$, where $E$ is the Young modulus of the metallic glass and $S$ is the stiffness ratio of sample $\kappa_{s}$ to the testing machine $S=\kappa_{s} / \kappa_{M}=\pi d^{2} E /\left(4 L \kappa_{M}\right)[19]$. 
The metallic glasses behave as an isotropic continuum solid on the macroscale; therefore, we consider the glassy phase in the framework of a continuous system. Before the construction of the strain distribution in a compressed sample, it is required to introduce a scaled variable $x(=\zeta / L) \epsilon$ $[0,1]$ (here, $L$ is the sample height and $\zeta$ is the original position at the length direction of the sample). After setting time as $t$, a differential equation is established as follows:

$$
\frac{4 M}{\pi d^{2}} U_{t t}=\sigma(0)-k U+k_{c} h^{2} U_{x x}-\sigma_{f}\left(U_{t}\right),
$$

where $\sigma(0)$ is the initial internal stress, which is equal to the yield stress, $\sigma_{R}$, and $\sigma_{f}(\dot{U})=\left(\sigma_{R}-A_{2} E\left(T-T_{R}\right) / T_{g}\right) /(1+$ $\left.A_{1} \dot{U}\right)$ [4], which represents the shear resistance along the shear plane (where $T$ is the environmental temperature, $T_{R}$ is the room temperature, $\sigma_{R}$ is the yield stress at room temperature, $T_{g}$ is the glass-transition temperature, and $A_{2}$ is a dimensionless constant determined to be 0.0106 for various BMGs). For typical Zr-based BMGs, the constant $A_{1}=5$ [5]. $d$ is the diameter of the sample. $M$ is the equivalent mass of the system, which consists of a metallic glass and a spring that represents the influence of the testing machine. $k=E / L(1+S)$, where $E$ is the Young modulus of the metallic glass and $S$ is the stiffness ratio of sample $\kappa_{s}$ to the testing machine $S=\kappa_{s} / \kappa_{M}=\pi d^{2} E /\left(4 L \kappa_{M}\right)[19]$.

The propagation of the shear bands can be considered as a wave propagating in homogeneous isotropic elastic body [20, 21]. To solve the partial differential equation (2), the strain evolution was traced during the deformation by analyzing the traveling wave transformation, $\xi=x-c t$, where $c$ is the speed of the traveling wave, $U(x, t)=U(\xi)$. Equation (2) can be translated into the following ordinary differential equation:

$$
\left(\frac{4 M c^{2}}{\pi d^{2}}-k_{c} h^{2}\right) U_{\xi \xi}+k U+\sigma_{f}\left(U_{\xi}\right)-\sigma(0)=0 .
$$

If $A_{1} c \dot{U}$ is small, substitute the damping term $\sigma_{f}(\dot{U})=$ $\left(\sigma_{R}-A_{2} E\left(T-T_{R}\right) / T_{g}\right) /\left(1-A_{1} c \dot{U}\right)$ by a perturbation item, $\left(\sigma_{R}-A_{2} E\left(T-T_{R}\right) / T_{g}\right)\left(1+\varepsilon A_{1} c \dot{U}+\varepsilon^{2} A_{1}^{2} c^{2} \dot{U}^{2}\right)$; then (3) can be rewritten into

$$
\begin{aligned}
& \left(\frac{4 M c^{2}}{\pi d^{2}}-k_{c} h^{2}\right) \ddot{U}+k U \\
& \quad=-\left(\sigma_{R}-\frac{A_{2} E\left(T-T_{R}\right)}{T_{g}}\right)\left(\varepsilon A_{1} c \dot{U}+\varepsilon^{2} A_{1}^{2} c^{2} \dot{U}^{2}\right) .
\end{aligned}
$$

The system (4) can be rewritten as

$$
\begin{aligned}
& \left(\frac{4 M c^{2}}{\pi d^{2}}-k_{c} h^{2}\right) \ddot{U}+k U \\
& +\left(\sigma_{R}-\frac{A_{2} E\left(T-T_{R}\right)}{T_{g}}\right) \varepsilon A_{1} c \dot{U} \\
& +\left(\sigma_{R}-\frac{A_{2} E\left(T-T_{R}\right)}{T_{g}}\right) \varepsilon^{2} A_{1}^{2} c^{2} \dot{U}^{2}=0 .
\end{aligned}
$$

Set $a=4 M c^{2} / \pi d^{2}-k_{c} h^{2}$; if $a>0$, set $\Omega_{0}=\sqrt{a / k}, t=\Omega_{0} \tau$, $U=b x$, where $b=a /\left(\sigma_{R}-A_{2} E\left(T-T_{R}\right) / T_{g}\right) A_{1}^{2} c^{2}$. By using the linear transformation $U=b x, t=\Omega_{0} \tau$, the original system is transformed into the following:

$$
\ddot{x}+x+Q \varepsilon \dot{x}+\varepsilon^{2} \dot{x}^{2}=0,
$$

where $Q=\left(\sigma_{R}-A_{2} E\left(T-T_{R}\right) / T_{g}\right) A_{1} c / k \Omega_{0}$. The system (6) has equivalent orbital with the original dynamics; then we analyze the stability of the system (5) [22]. Set $x=x_{1}, \dot{x}=x_{2}$; the system (6) is transformed into

$$
\begin{aligned}
& \dot{x}_{1}=x_{2}, \\
& \dot{x}_{2}=-x_{1}-Q \varepsilon x_{2}-\varepsilon^{2} x_{2}^{2} .
\end{aligned}
$$

The equilibrium point is $O(0,0)$; the corresponding derived operator is

$$
D f(0,0, \varepsilon)=\left[\begin{array}{cc}
0 & 1 \\
-1 & -Q \varepsilon
\end{array}\right] \text {. }
$$

If $\varepsilon=0, D f(0,0,0)$ has a pair of pure imaginary characteristic roots, $\pm i$. The equilibrium point $O(0,0)$ is non-hyperbola equilibrium point, and the stability of the equilibrium point depends on the higher-order nonlinear term requiring further analysis. We firstly analyze the characteristics of the phase trajectory of the system (7) nearby $\varepsilon=0(\varepsilon \neq 0)$.

(a) If $Q>0$ st. $|Q \varepsilon|<2$,

(1) If $\varepsilon<0$, the characteristic roots of the derived operator at the equilibrium point $O(0,0)$ are

$$
\lambda_{1,2}=-\frac{Q \varepsilon}{2} \pm \frac{\sqrt{(Q \varepsilon)^{2}-4}}{2}
$$

and the characteristic roots $\lambda_{1}, \lambda_{2}$ are conjugate complex roots, with positive real part. The equilibrium point $O(0,0)$ is an unstable focus.

(2) If $\varepsilon>0$, the characteristic roots $\lambda_{1}, \lambda_{2}$ are conjugate complex roots, with negative real part. The equilibrium point $O(0,0)$ is a stable focus.

As the value of $\varepsilon$ goes through $\varepsilon=0$ increasing from negative to positive, the number of the equilibrium points is unchanged, while the stability changes from unstable to stable.

(b) If $Q<0$ st. $|Q \varepsilon|<2$,

(1) If $\varepsilon<0$, the characteristic roots $\lambda_{1}, \lambda_{2}$ are conjugate complex roots, with negative real part. The equilibrium point $O(0,0)$ is a stable focus.

(2) If $\varepsilon>0$, the characteristic roots $\lambda_{1}, \lambda_{2}$ are conjugate complex roots, with positive real part. The equilibrium point $O(0,0)$ is an unstable focus.

Note that, at $\varepsilon=0$, the characteristic roots of the derived operator are a pair of pure imaginary characteristic roots, $\pm i$. In fact there is limit cycle at $\varepsilon=0$ corresponding to a periodic solution. 
TABLE 1: The parameters for the metallic glass $\mathrm{Cu}_{50} \mathrm{Zr}_{45} \mathrm{Ti}_{5}$ during the plastic deformation.

\begin{tabular}{lccccccccc}
\hline Parameter & $d$ & $L$ & $T_{R}$ & $T_{g}$ & $A_{1}$ & $A_{2}$ & $E$ & $M$ & $\sigma_{R}$ \\
\hline Value & 2 & 4 & 273 & 643 & 5 & 0.0106 & 80 & 0.0829 & 1742 \\
Unit & $\mathrm{mm}$ & $\mathrm{mm}$ & $\mathrm{K}$ & $\mathrm{K}$ & 1 & 1 & $\mathrm{GPa}$ & $\mathrm{g}$ & $\mathrm{MPa}$ \\
\hline
\end{tabular}

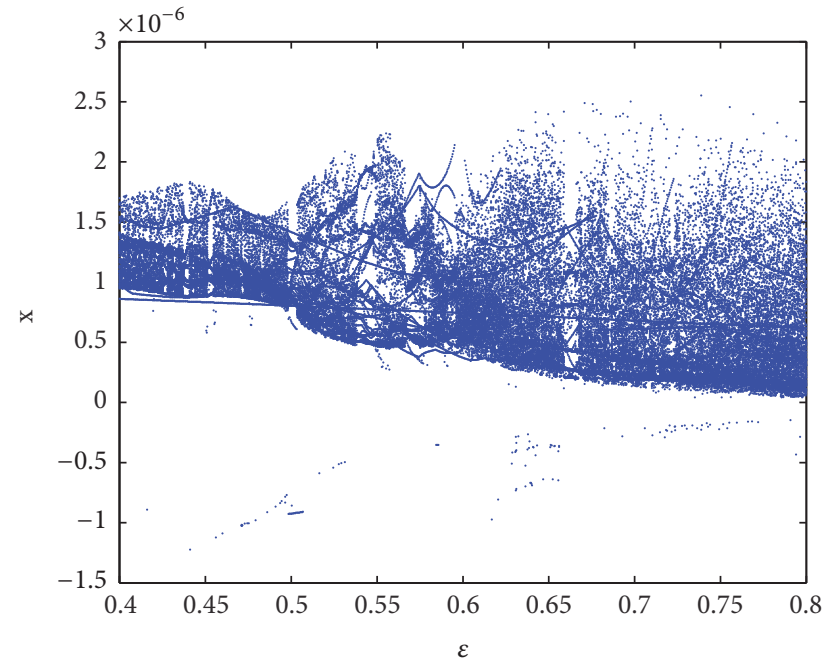

FIGURE 2: The bifurcation diagram of the system (6) for the parameter $\varepsilon$ range in $[0.4,0.8], Q=1$.

\section{Numerical Simulation}

The numerical simulation was conducted based on the system (6). The parameters in the experiment are shown in Table 1. The solution of the system varies with the perturbation parameter $\varepsilon$, and the phase diagram for different values of $\varepsilon$ is shown in Figure 2 for $Q=1$. The time history, the phase diagram, spectrum, and the Poincare section of the system (6) for $Q=1$ are shown in Figures 3-7. For the parameter $\varepsilon=0$, there is periodic solution corresponding to the limit cycle in the phase diagram (Figure 3). As $\varepsilon$ increases, the solution of the system manifests period-doubling oscillation at $\varepsilon=0.55$ (see Figures 2 and 4). For $\varepsilon=0.653$, the solution of the system is quasi-periodic from the phase diagram (see Figure 6). The various frequency in the oscillation solution also indicates that there exists multiscale behavior in the serrated flow dynamics [9]. Note that $Q=\left(\sigma_{R}-A_{2} E\left(T-T_{R}\right) / T_{g}\right) A_{1} c / k \Omega_{0}$, $Q>0$, means $T<T_{g} \sigma_{R} / A_{2} E+T_{R}$; if the temperature is lower than a certain value, the system can evolve to a stable state at a certain condition (see Figure 3). This is corresponding to the conclusion that the serrated flow is self-organized to a critical state at low temperatures $[1,8]$. There is a transition from a criticality state to a limit cycle dominated by the inertia effect, which is similar to the phenomenon in a dynamic model for the tuned criticality in stick-slip friction during metal cutting [23].

For $Q=-1$, the time history, the phase diagram, spectrum, and the Poincaré section of the system (6) are shown in Figures 8 and 9. For the parameter $\varepsilon=0.05385$, there is unstable solution for the system (see Figure 8). For the parameter $\varepsilon=0.06885$, the oscillation solution has nonuniform structure (See Figure 9), which indicates the motion of the shear bands is slow-fast system at this case. In fact, the original serrated flow signal (such as the stress-time curve) shows smaller serration followed by a large stress drop. Note that $Q=\left(\sigma_{R}-A_{2} E\left(T-T_{R}\right) / T_{g}\right) A_{1} c / k \Omega_{0}, Q<0$, means $T>T_{g} \sigma_{R} / A_{2} E+T_{R}$. It suggests that if the temperature is higher than a certain value, the system is unstable, which is in accordance with our previous result about the time series analysis based on the experimental data [8].

Moreover, the numerical simulation based on (2) is resolved with $\sigma_{f}(\dot{U})=\left(\sigma_{R}-A_{2} E\left(T-T_{R}\right) / T_{g}\right)\left(1+\varepsilon A_{1} c \dot{U}+\right.$ $\left.\varepsilon^{2} A_{1}^{2} c^{2} \dot{U}^{2}\right)$. The periodic boundary conditions $U(x, 0)=0$, $U(x, 0)=U(x, L), U_{t}(x, 0)=v_{0}$. Considering, at $t=0$, the sliding speed of each block is unknown, set $v_{0}$, a random number which is smaller than $v$. There are different sizes of sliding events, and some of them are shown in Figures $10(\mathrm{a})-10$ (c) in the form of the sliding velocity $\dot{U}$ as a function of positions $i$ and time $t$.

Based on the numerical results, the statistics of the sliding speed of the i-th blocks, $\dot{U}_{i}$ (here $\dot{U}_{i}$ is denoted by $s$ for convenience) at the temperature of $173 \mathrm{~K}$, show a powerlaw distribution with a fitting exponent of $\alpha=-2.63$ [see Figure $10(\mathrm{~d})]$. Noting that $\Delta \sigma=k(s-v) \Delta t$, the stress drops also behave as a power-law distribution for larger $\varepsilon=$ 0.03001975 at the temperature of $293 \mathrm{~K}$, which means the selforganized critical behavior in the plastic fracture.

Then we present how the self-organizing criticality phenomenon develops in plastic dynamics. The motion of shear bands is the main mechanism dominating the plastic deformation for BMGs. Before the shear banding process, an elastic strain field develops in the glassy phase [24]. The size of elastic strain field is much larger than the interspace between neighboring shear blocks. Then the neighboring elastic strain fields must interfere with each other during the shear banding process. During the motion of the shear bands, it accompanies the accumulation and the release of the energy. For larger disturbed parameter, there is stronger interference between the neighboring elastic strain fields. The elastic strain field cannot be totally relaxed in limited time, and then the new shear bands are formed in the location of the unrelaxed elastic strain fields. The overlap of the elastic strain fields results in a hierarchy of length scales, leading to the selforganizing criticality behavior [1]. The larger perturbation parameter reflects the stronger mutual interaction between the elastic strain fields, which is corresponding to ductile BMGs. This is quite consistent with the result based on the experimental data, which showed that the plastic dynamics manifested as self-organized critical state for more ductile BMGs [5].

For a given $T=293 K$, the sliding speed of the shear bands is lower for a smaller $\varepsilon=0.025$ (Figures 10(a) and 10 (b)). For a given $\varepsilon=0.03001975$, it suggests that the shear 


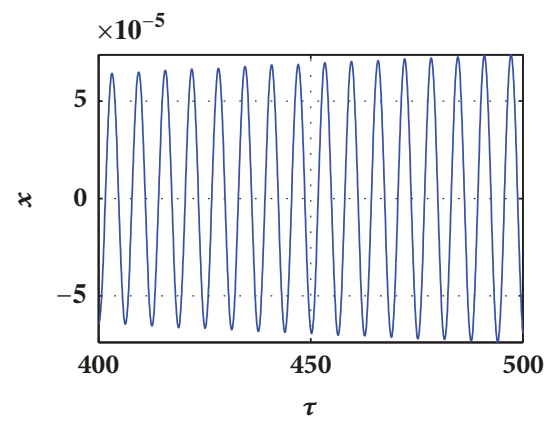

(a)

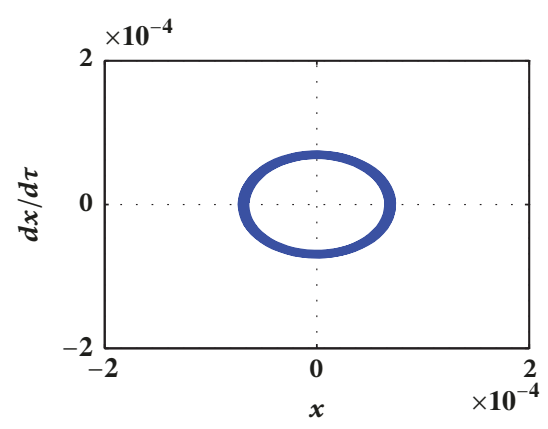

(b)

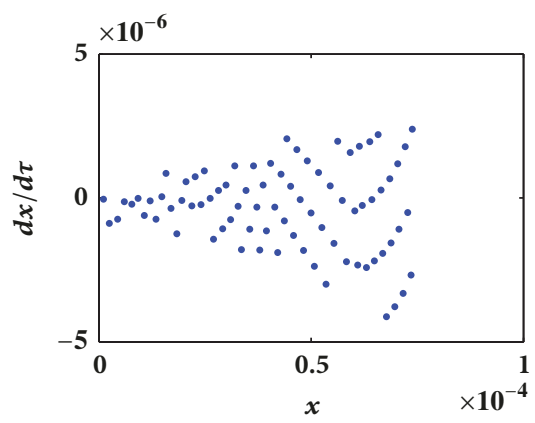

(d)

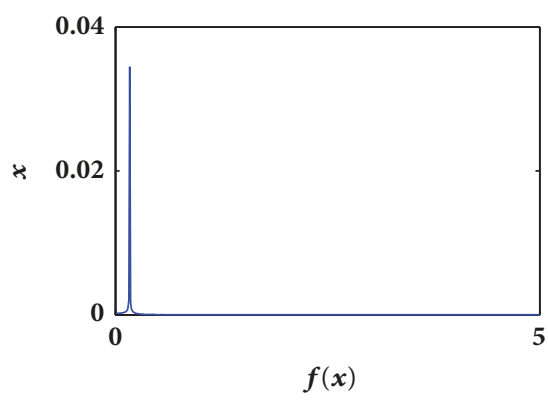

(c)

FIgURE 3: The time history, the phase diagram, spectrum, and the Poincare section of the system (6) for $\varepsilon=10^{-5}, Q=1$.

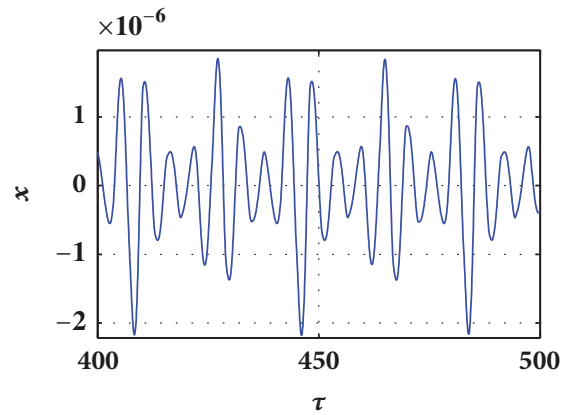

(a)

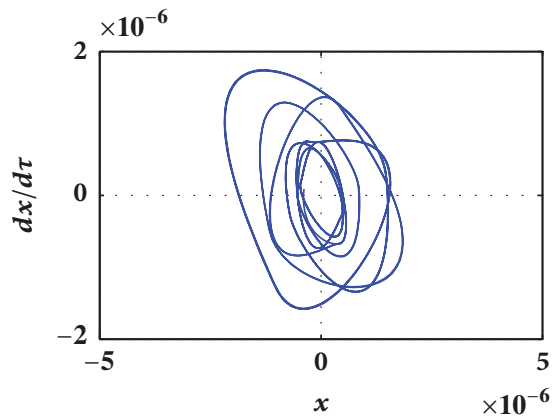

(b)

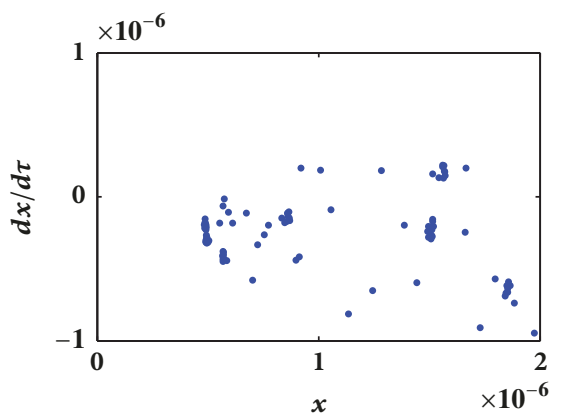

(d)

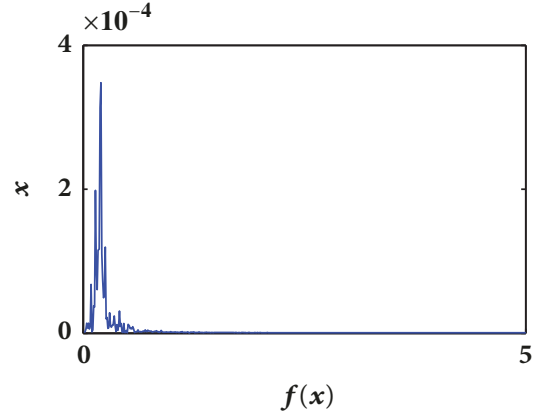

(c)

FuRE 4: The time history, the phase diagram, spectrum, and the Poincaré section of the system (6) for $\varepsilon=0.55, Q=1$.

bands slide with a lower sliding speed for a smaller $T=$ $173 \mathrm{~K}$ (Figures $10(\mathrm{~b})$ and $10(\mathrm{c})$ ). The most distinctive feature in Figures $10(\mathrm{a})-10(\mathrm{c})$ is that there is oscillation in sliding speed which is corresponding to the serrated flow in the stresstime curves $[1,8]$. From the numerical simulation, for smaller $\varepsilon=0.025$, the sliding speed fluctuates periodically. There are larger and irregular sliding events when the parameters $\varepsilon$ is large, and the sliding of the shear bands will be more complex.

The complexity of the system is investigated considering the influence of the disturbed parameters $\varepsilon$ and the 


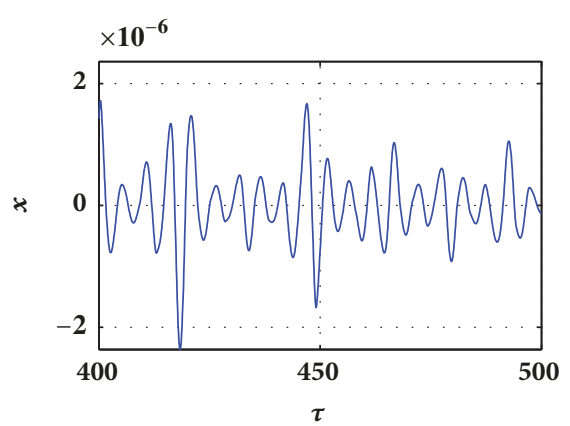

(a)

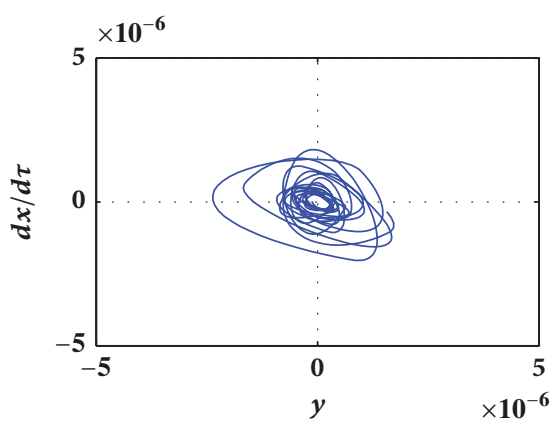

(b)

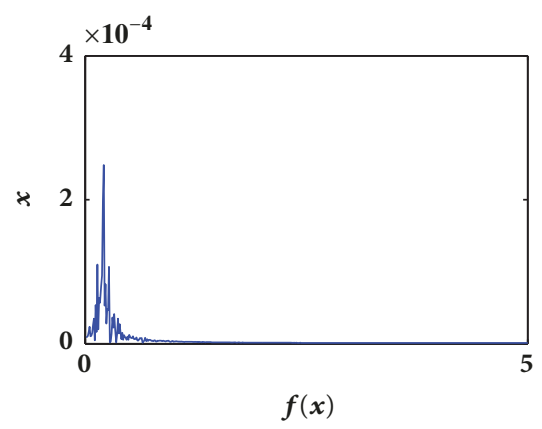

(c)

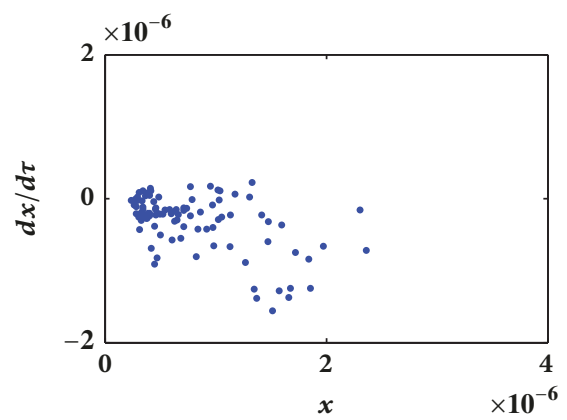

(d)

Figure 5: The time history, the phase diagram, spectrum, and the Poincaré section of the system (6) for $\varepsilon=0.6529, Q=1$.

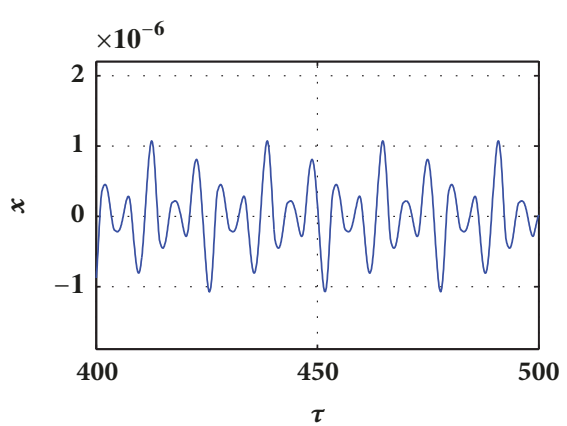

(a)

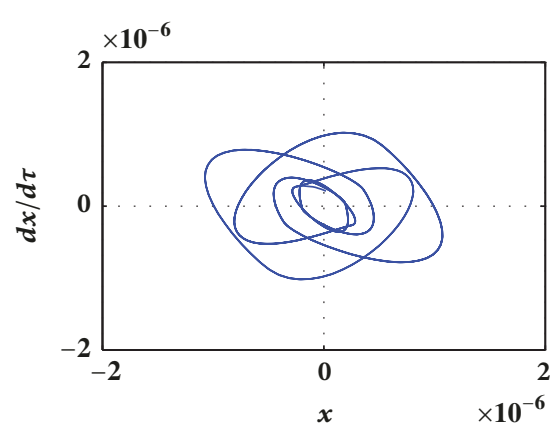

(b)

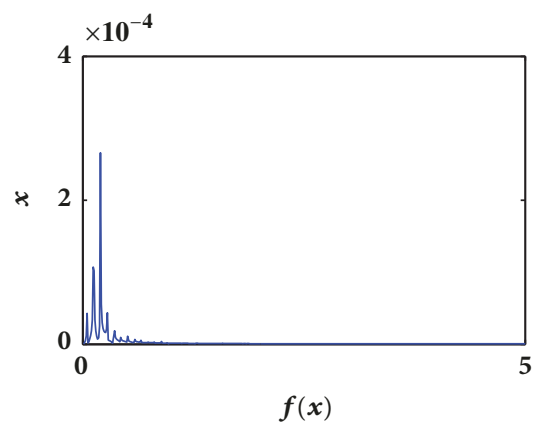

(c)

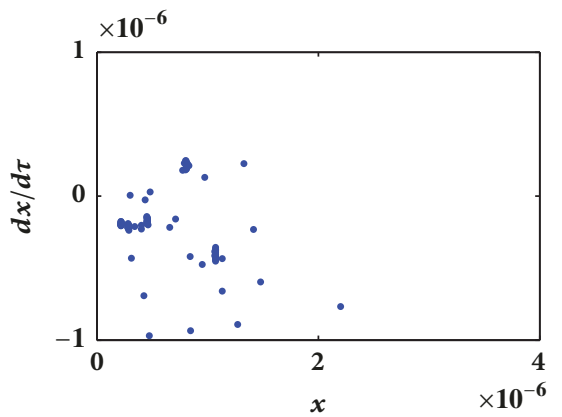

(d)

Figure 6: The time history, the phase diagram, spectrum, and the Poincaré section of the system (6) for $\varepsilon=0.653, Q=1$.

temperature. The larger disturbed parameter induced more interference in the shear banding process, which increases the complexity of the system. To characterize the complexity of the system, we calculate the approximate entropy (ApEn) [25] of $\left\{d U_{i} / d t, i=1,2, \ldots, N\right\}$ based on the numerical results of system (2), where $N=2601$, and the parameters $r=0.1, m=$ 2. The value of ApEn increases as the disturbance parameter $\varepsilon$ is increasing, and the calculated value of ApEn is shown as 


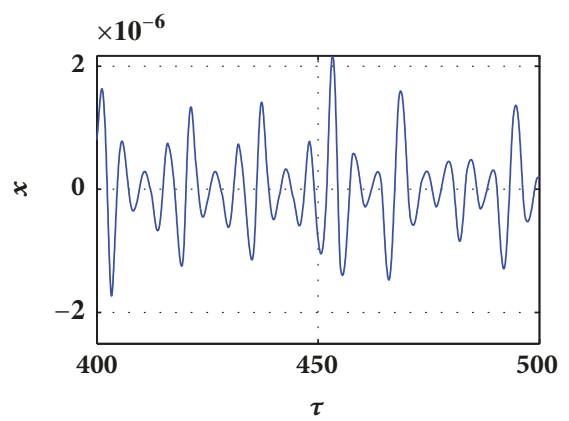

(a)

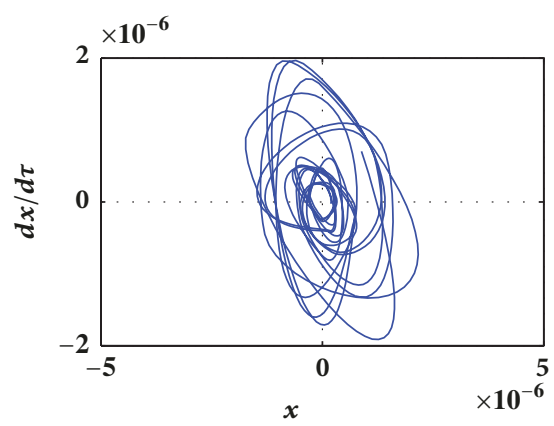

(b)

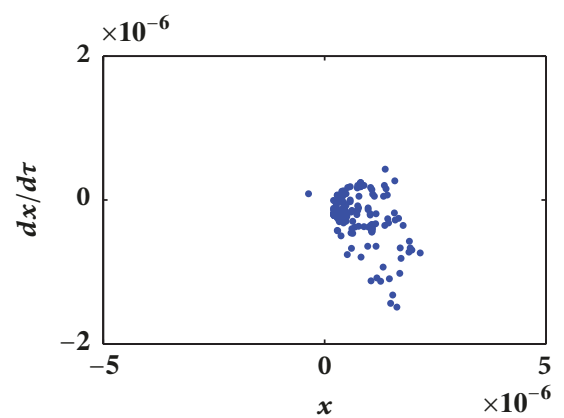

(d)

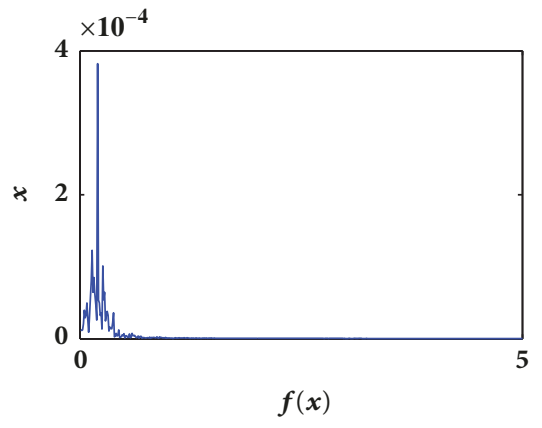

(c)

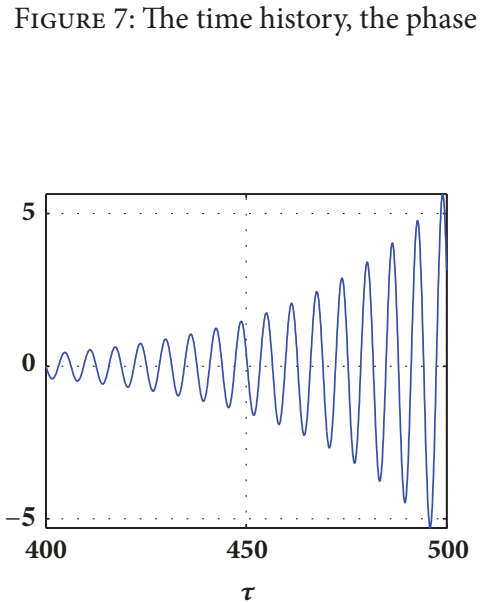

(a)

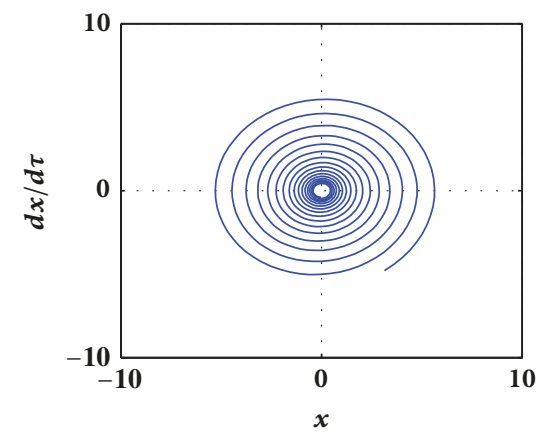

(b)

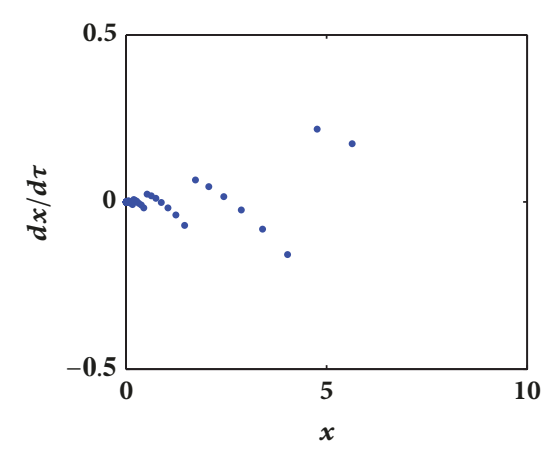

(d)

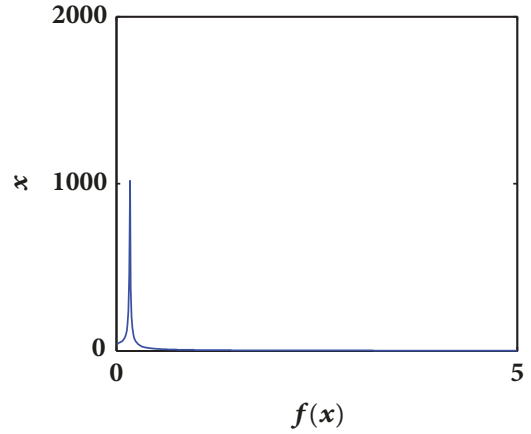

(c)

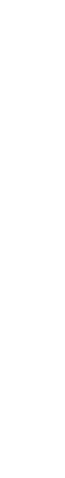

Figure 8: The time history, the phase diagram, spectrum, and the Poincaré section of the system (6) for $\varepsilon=0.05385, Q=-1$. 


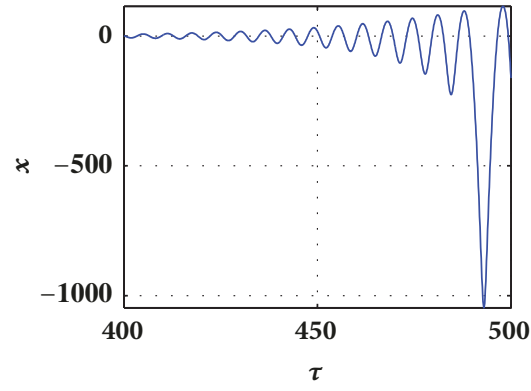

(a)

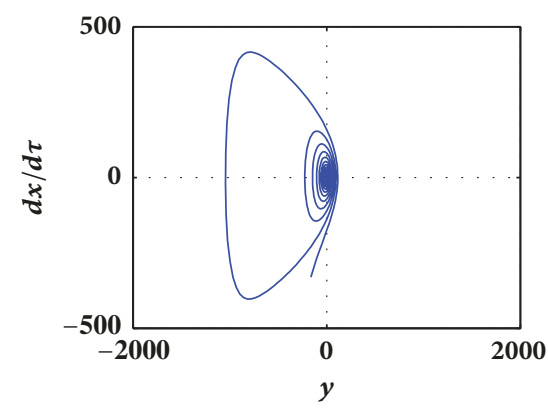

(b)

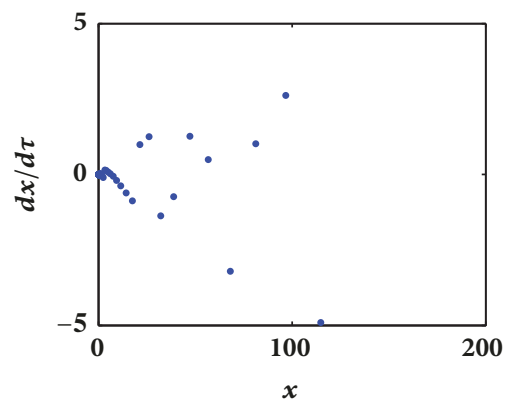

(d)

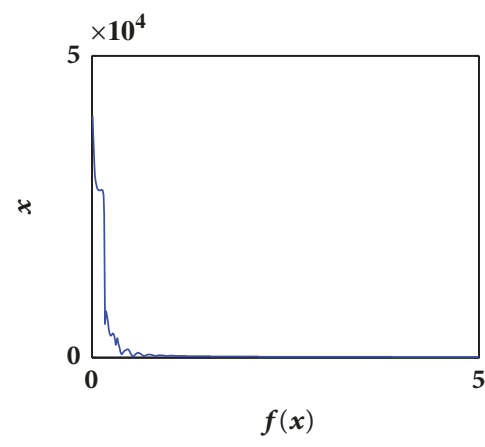

(c)

FIGURE 9: The time history, the phase diagram, spectrum, and the Poincaré section of the system (6) for $\varepsilon=0.06885, Q=-1$.

a function of temperature presented in Figure 11, where the parameter $\varepsilon \in[0.02,0.03]$. Larger value of ApEn for larger disturbance parameter suggests the motion of the shear bands exhibits a high complexity. In addition, the values of ApEn are also calculated to evaluate the complexity for different temperatures [see Table 2], which suggests that at the lower temperature the system is with less complexity. This result is consistent with the above analysis that the solution is more stable at lower temperature.

\section{Multiscale Analysis}

The method of multiple scales [26] is developed to analyze the nonlinear shear banding system (4). Set $T_{n}=\varepsilon^{n} \xi(n=0,1,2)$, $D_{n} \equiv \partial / \partial T_{n}$,

$$
\begin{aligned}
\frac{d}{d \xi} & =D_{0}+\varepsilon D_{1}+\varepsilon^{2} D_{2} \\
\frac{d^{2}}{d \xi^{2}} & =D_{0}^{2}+2 \varepsilon D_{0} D_{1}+\varepsilon^{2}\left(D_{1}^{2}+2 D_{0} D_{2}\right)
\end{aligned}
$$

we seek an approximate solution of (4) in the form

$$
\begin{aligned}
U= & U_{0}\left(T_{0}, T_{1}, T_{2}\right)+\varepsilon U_{1}\left(T_{0}, T_{1}, T_{2}\right) \\
& +\varepsilon^{2} U_{2}\left(T_{0}, T_{1}, T_{2}\right) .
\end{aligned}
$$

Substituting (10) and (11) into (4) yields

$$
\begin{aligned}
& \left(\frac{4 M c^{2}}{\pi d^{2}}-k_{c} h^{2}\right)\left[D_{0}^{2}+2 \varepsilon D_{0} D_{1}+\varepsilon^{2}\left(D_{1}^{2}+2 D_{0} D_{2}\right)\right] \\
& \cdot\left(U_{0}+\varepsilon U_{1}+\varepsilon^{2} U_{2}\right)+k\left(U_{0}+\varepsilon U_{1}+\varepsilon^{2} U_{2}\right) \\
& =-\left(\sigma_{R}-\frac{A_{1} E\left(T-T_{R}\right)}{T_{g}}\right)\left[\varepsilon A _ { 1 } c \left(D_{0}+\varepsilon D_{1}\right.\right. \\
& \left.+\varepsilon^{2} D_{2}\right)\left(U_{0}+\varepsilon U_{1}+\varepsilon^{2} U_{2}\right) \\
& +\varepsilon^{2} A_{1}^{2} c^{2}\left(\left(D_{0}+\varepsilon D_{1}+\varepsilon^{2} D_{2}\right)\right. \\
& \left.\left.\cdot\left(U_{0}+\varepsilon U_{1}+\varepsilon^{2} U_{2}\right)\right)^{2}\right] .
\end{aligned}
$$

For convenience, set $4 M c^{2} \varepsilon^{2} / \pi d^{2}-k_{c} h^{2}=G, k / G=\omega_{0}^{2}$, $\left(\sigma_{R}-A_{2} E\left(T-T_{R}\right) / T_{g}\right) / G=p, A_{1} c=q$; for $\varepsilon=0$, we obtain the linear equations,

$$
\begin{aligned}
D_{0}^{2} U_{0}+\omega_{0}^{2} U_{0}= & 0 \\
D_{0}^{2} U_{1}+\omega_{0}^{2} U_{1}= & -p q D_{0} U_{0}-2 D_{0} D_{1} U_{0} \\
D_{0}^{2} U_{2}+\omega_{0}^{2} U_{2}= & -2 D_{0} D_{1} U_{1}-\left(D_{1}^{2}+2 D_{0} D_{2}\right) U_{0} \\
& -p q\left(D_{0} U_{1}+D_{1} U_{0}\right) \\
& -p q^{2}\left(D_{0} U_{0}\right)^{2}
\end{aligned}
$$




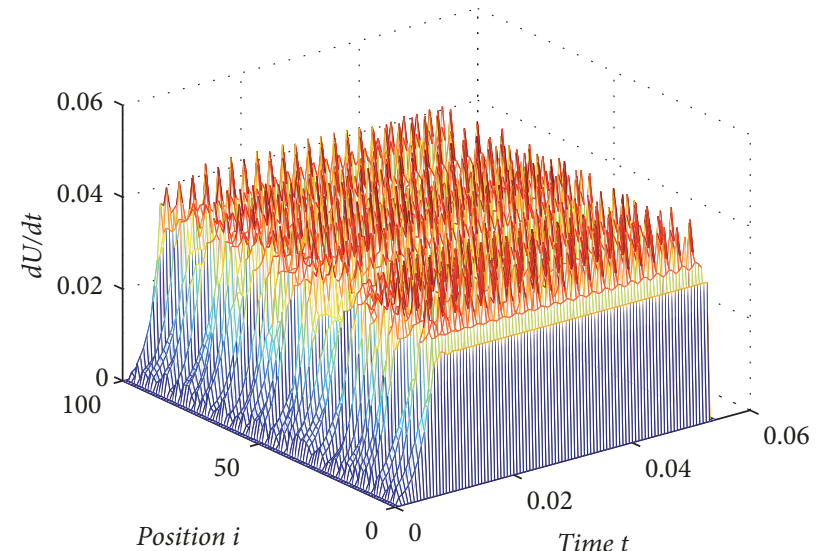

(a)

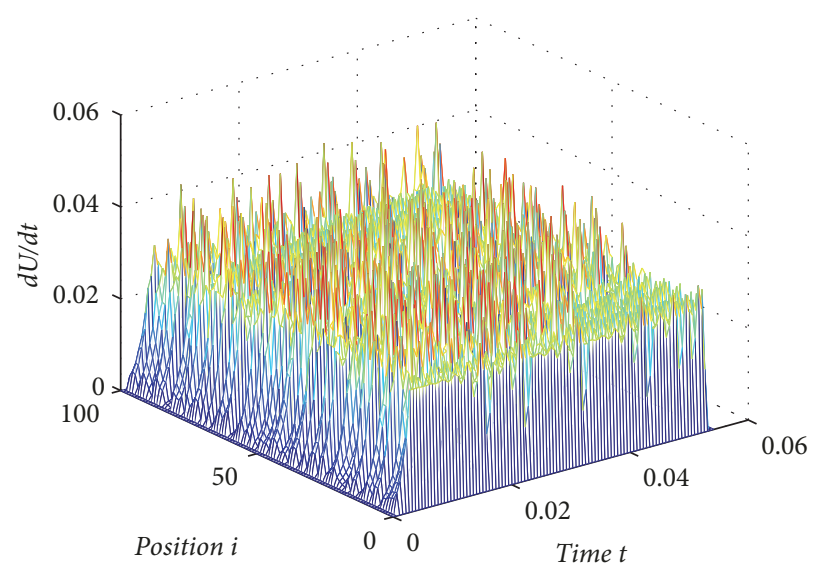

(c)

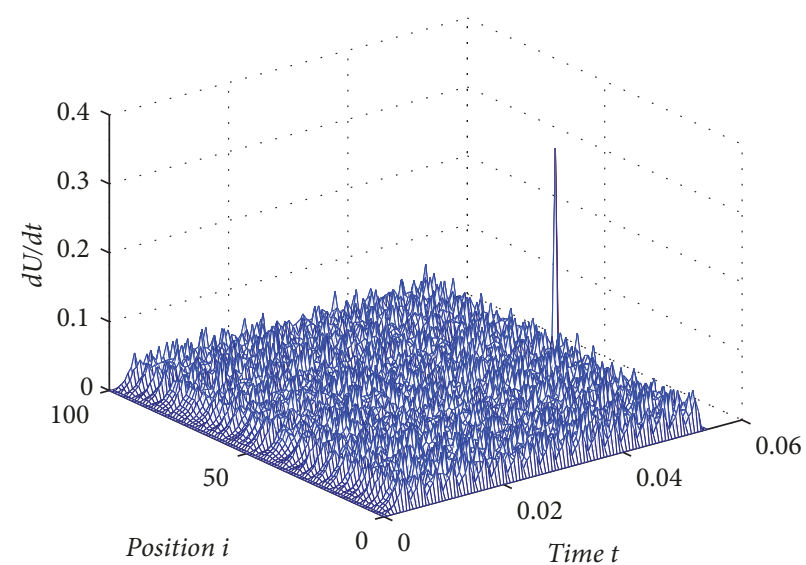

(b)

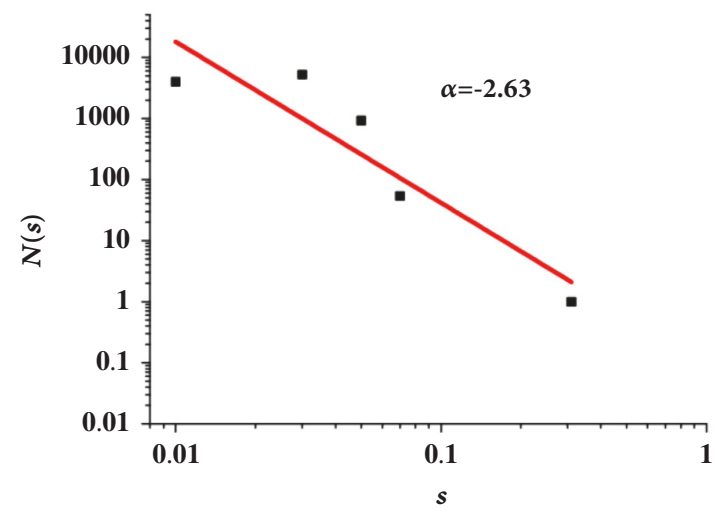

(d)

FIGURE 10: Numerical simulation at different temperatures $T$ and perturbation parameters $\varepsilon$. (a) $T=293 K, \varepsilon=0.025$; (b) $T=293 K$, $\varepsilon=$ 0.03001975 ; (c) $T=173 K, \varepsilon=0.03001975$; (d) the distribution $N$ vs. the sliding speed, $d U / d t$, of each block at $T=293 K, \varepsilon=0.03001975$, where $N()$ represents the number of blocks with sliding speed, $d U / d t . v_{0}=0.00001, c=340$, and the other parameters used here are listed in Table 1.

TABLE 2: The ApEn of $\left\{d U_{i} / d t, i=1,2, \cdots, N\right\}$ for different temperatures.

\begin{tabular}{lcccc}
\hline$T$ & 173 & 213 & 253 & 293 \\
\hline ApEn & 1.5559 & 1.6267 & 1.6709 & 1.6798 \\
\hline
\end{tabular}

The solution of (14) can be written as

$$
U_{0}=A\left(T_{1}, T_{2}\right) e^{i \omega_{0} T_{0}}+\bar{A}\left(T_{1}, T_{2}\right) e^{-i \omega_{0} T_{0}}
$$

where $\omega_{0}=\sqrt{k / G}, A$ is the undetermined complex function, and $\bar{A}$ is the complex conjugate of $A$.

Substituting (17) into (15), it can be deduced that

$$
k_{c} h^{2} D_{0}^{2} U_{1}-k U_{1}=-\left(2 D_{1} A+p q A\right) i \omega_{0} e^{i \omega_{0} T_{0}}+c c
$$

where $c c$ is the complex conjugate of the preceding terms. Eliminating the secular term in (18), we obtain

$$
2 D_{1} A+p q A=0
$$

which means the amplitude $A$ varies with $T_{1}$. From (18) we get

$$
U_{1}=A\left(T_{1}, T_{2}\right) e^{i \omega_{0} T_{0}}+\bar{A}\left(T_{1}, T_{2}\right) e^{-i \omega_{0} T_{0}} .
$$

Substituting (17) and (20) into (16) yields

$$
\begin{gathered}
k_{c} h^{2} D_{0}^{2} U_{2}-k U_{2}=-\left[2 i \omega_{0} D_{1} A+D_{1}^{2} A+2 D_{2} A i \omega_{0}\right. \\
\left.+p q\left(i \omega_{0} A+D_{1} A\right)\right] e^{i \omega_{0} T_{0}}+p q^{2} \omega_{0}^{2} A^{2} e^{2 i \omega_{0} T_{0}}+c c .
\end{gathered}
$$

Eliminating the secular term in (21), we have

$$
2 i \omega_{0} D_{1} A+D_{1}^{2} A+2 D_{2} A i \omega_{0}+p q\left(i \omega_{0} A+D_{1} A\right)=0 \text {. }
$$

The solution of (21) can be expressed as

$$
U_{2}=-\frac{p q^{2} A^{2}}{3} e^{2 i \omega_{0} T_{0}}+c c .
$$




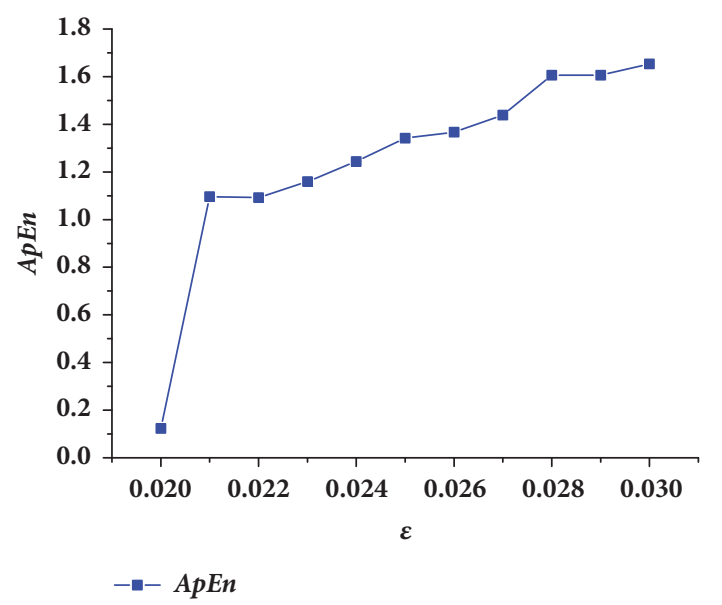

Figure 11: The ApEn of $\left\{d U_{i} / d t, i=1,2, \ldots, N\right\}$ based on the system (2) for different disturbance parameter $\varepsilon$. The parameters used here are listed in Table 1.

Equation (22) determines the amplitude $A$ varying with $T_{2}$. Considering

$$
\frac{d A}{d \xi}=D_{0} A+\varepsilon D_{1} A+\varepsilon^{2} D_{2} A,
$$

where $D_{0} A=0, D_{1} A$, and $D_{2} A$ are determined by (19) and (22), respectively, we can deduce the differential equation $A$ satisfying

$$
\frac{d A}{d \xi}=-\varepsilon \frac{p q A}{2}-\varepsilon^{2} \frac{p^{2} q^{2} A}{8 \omega_{0}} i
$$

Set

$$
A(\xi)=\frac{1}{2} a(t) e^{i \theta(\xi)},
$$

From (25) we get

$$
\begin{aligned}
& \dot{a}=-\frac{\varepsilon p q}{2} a, \\
& \dot{\theta}=-\frac{\varepsilon^{2} p^{2} q^{2}}{8 \omega_{0}} .
\end{aligned}
$$

It can be deduced that

$$
\begin{aligned}
& a=e^{-(\varepsilon p q / 2) \xi}+c_{1}, \\
& \theta=-\frac{\varepsilon^{2} p^{2} q^{2}}{8 \omega_{0}} \xi+c_{2} .
\end{aligned}
$$

where $c_{1}, c_{2}$ are constants. Set $\alpha=\varepsilon p q / 2, \beta=\varepsilon^{2} p^{2} q^{2} / 8 \omega_{0}$; then we get

$$
A(\xi)=\frac{1}{2}\left(e^{-\alpha \xi}+c_{1}\right) e^{i\left(-\beta \xi+c_{2}\right)} .
$$

Substituting (17), (20), and (23) into (12) yields the solution

$$
\begin{aligned}
U= & A\left(T_{1}, T_{2}\right) e^{i \omega_{0} T_{0}}+\varepsilon A\left(T_{1}, T_{2}\right) e^{i \omega_{0} T_{0}} \\
& -\varepsilon^{2} \frac{p q^{2} A^{2}}{3} e^{2 i \omega_{0} T_{0}}+c c .
\end{aligned}
$$

The approximate solution of (4) can be expressed as

$$
\begin{aligned}
U(\xi)= & \left(e^{-\alpha \xi}+c_{1}\right) \cos \left(\xi \omega_{0}-\beta \xi+c_{2}\right) \\
& +\varepsilon\left(e^{-\alpha \xi}+c_{1}\right) \cos \left(\xi \omega_{0}-\beta \xi+c_{2}\right) \\
& -\varepsilon^{2} \frac{p q^{2}}{6}\left(e^{-\alpha \xi}+c_{1}\right)^{2} \cos \left(2 \xi \omega_{0}-2 \beta \xi+2 c_{2}\right)
\end{aligned}
$$

To investigate the numerical solution $U(\xi)$ qualitatively, for convenience, set the constant $c_{1}=0, c_{2}=0$; the curve of $U(\xi)$ at the temperature $T=293 \mathrm{~K}$ for perturbation parameter $\varepsilon=10^{-8}$ is shown in Figure 12(a), and the curve for perturbation parameter $\varepsilon=10^{-7}$ is shown in Figure 12(b). It manifests that the system is unstable in the form of oscillation for smaller perturbation $\varepsilon=10^{-8}$, while it is stable for larger perturbation $\varepsilon=10^{-7}$. The value of the perturbation parameter $\varepsilon$ can be considered as the strength of the interaction between the shear bands. If $\varepsilon$ is in this small zone, the system evolves from unstable to stable state as $\varepsilon$ increases; this is consistent with the conclusion based on the experimental data analysis that the system is in unstable chaotic state for the weaker interaction between the shear bands corresponding to the brittle BMGs [5].

In addition, we investigate the approximate solution of the system (4) for different temperatures. Choose the parameter $\varepsilon=5 \times 10^{-8}$; the curves of $U(\xi)$ at the temperatures $T=$ $293 \mathrm{~K}$ and $T=173 \mathrm{~K}$ are shown in Figures 13(a) and 13(b). Zooming in on the two curves in Figure 13(c), it can be seen that the amplitude of solution decreases with the decreasing of temperature. The oscillation of the solution becomes weaker at lower temperature of $173 \mathrm{~K}$. It shows that the decreasing of the temperature can reduce the instability. This result is in accordance with our previous work based on the experimental data analysis. The serration in the stressstrain curves is smaller and the serrated flow dynamics is more stable at lower temperature [8]. The fractal dimension of the stress rate signal ranges from 1.22 to 1.72 with decreasing temperature and a larger shear-branching rate occurs at lower temperature. As the temperature changes, there is a temporal scale free behavior in the serrated flow [27], corresponding to the self-organized critical state.

\section{Conclusion and Discussion}

The system containing a series of sliding blocks has complex nonlinear dynamical behavior, which occurred in, for example, earthquake [28-30], slope forecasting, and friction experiment of rock mass [31-34]. The motion of an elastic string everywhere in contact with a frictional surface was constructed to explore the earthquake mechanism by Burridge and Knopoff in 1967 [35]. In this paper, a spatiotemporal dynamical model considering the temperature is proposed for describing the plastic deformation in bulk metal glass. The stability analysis, the numerical simulation, and multiscale analysis are applied to investigate the complex motions of the shear sliding blocks. The stability analysis shows that the shear slipping displacement system can evolve from stable to unstable with the parameter varying, and there is limit 


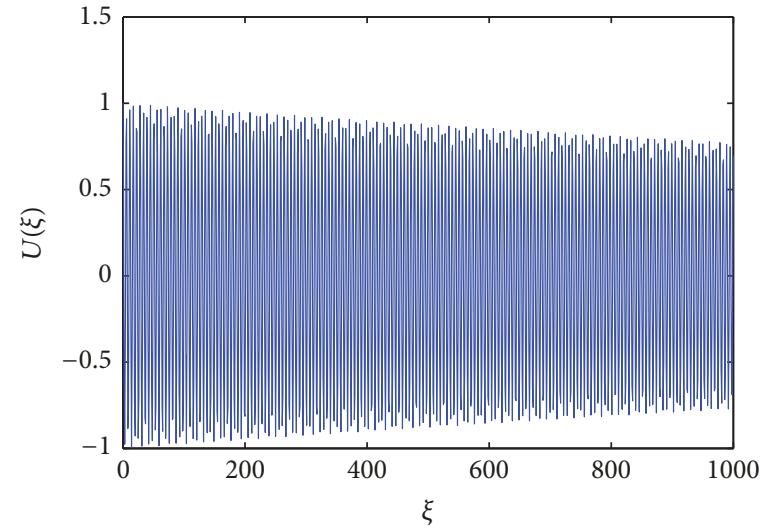

(a)

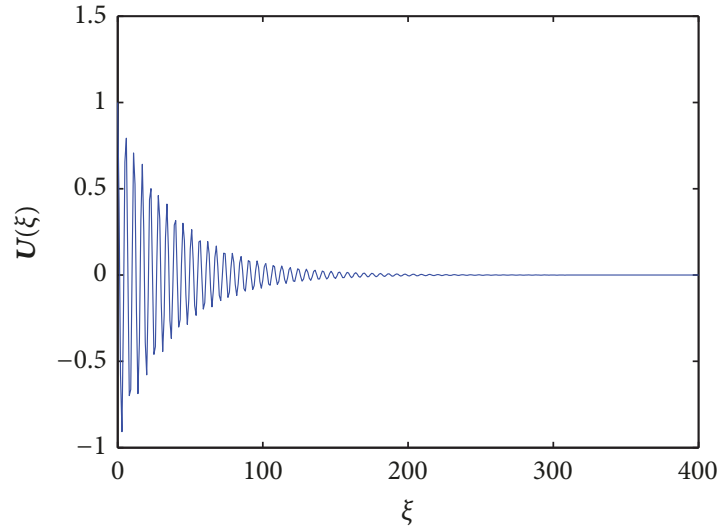

(b)

Figure 12: The curve of $U(\xi)$ at the temperature $T=293 \mathrm{~K}$ for perturbation parameters (a) $\varepsilon=10^{-8}$ and (b) $\varepsilon=10^{-7}$.

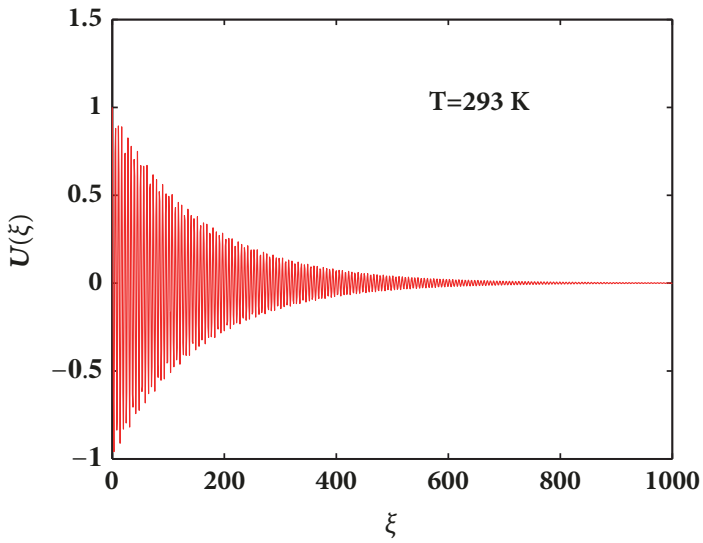

(a)

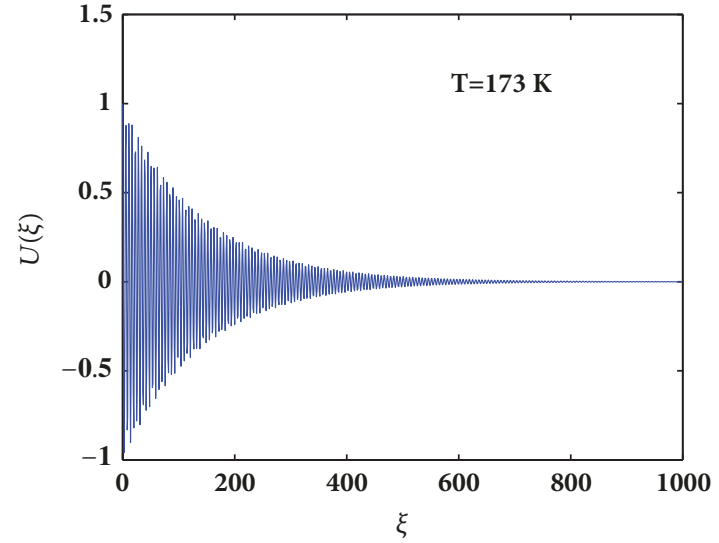

(b)

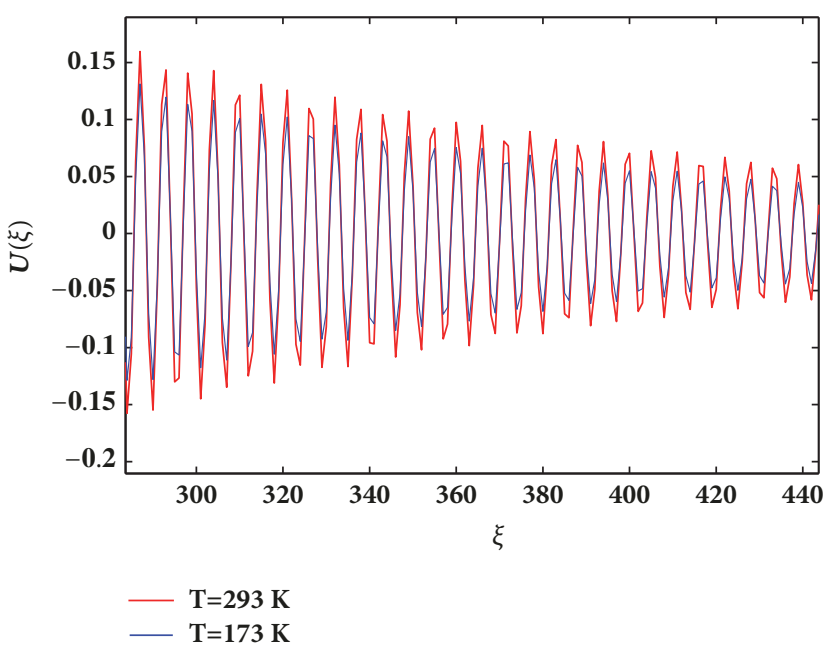

(c)

Figure 13: The curve of $U(\xi)$ at the temperatures (a) $T=293 \mathrm{~K}$ and (b) $T=173 \mathrm{~K}$ for perturbation parameter $\varepsilon=5 \times 10^{-8}$. (c) The difference of the solution at different temperatures. 
cycle at a transition stage. It presents theoretical support for explaining the transition from unstable states to stable states in the plastic dynamic system under different condition $[8,9]$.

Furthermore, we find that the system is unstable at larger perturbation parameter, which is corresponding to the stronger interference between the multiple shear bands, while, for the smaller perturbation parameter, there exists a stable state accompanied by weaker interaction between the multiple shear bands. Meanwhile, the numerical simulation shows that, at larger $\varepsilon$, the sliding speed of the shear bands is larger, and the solution is less regular. There is power law distribution during the motion for certain condition. The value of ApEn is calculated to measure the complexity of the system, and it suggests that larger disturbance makes the motion of the shear bands exhibit a higher complexity. This result provides clear theoretical explanation for the conclusion that the system is self-organized to a critical state for ductile BMGs [5], noting that the perturbation parameter reflects the degree of ductility.

In addition, based on the multiscale analysis, the influence of the temperature is investigated in the dynamical model. It suggests that the amplitude of the solution reduces with the decreasing of the temperature, which is consistent with the experimental data analysis that the serration in the stress-strain curves is smaller at lower temperature [8]. As temperature decreases, the system evolves to a more stable state, which is consistent with our previous experimental results $[8,27]$.

In conclusion, with the varying of the parameters, the spatiotemporal model manifests complex dynamical behaviors. The results are corresponding with the data analysis based on the experiment involving fractal $[27,36]$, chaos, selforganized critical state $[5,8]$, and the scaling behavior [27]. The motion of the shear bands is illustrated by the theoretical analysis and numerical simulation based on dynamical model, providing a fresh approach to the study of plastic deformation of bulk metallic glasses.

\section{Data Availability}

There is no experimental data used in this study.

\section{Conflicts of Interest}

The authors declare that they have no conflicts of interest.

\section{Acknowledgments}

This work was supported by the National Natural Science Foundation of China Grants (No. 11701521) and China Postdoctoral Science Foundation (No. 2018M632790). C. Chen very much appreciates experimental support from Professor G. Wang in Laboratory for Microstructures of Shanghai University.

\section{References}

[1] J. L. Ren, C. Chen, G. Wang, N. Mattern, and J. Eckert, "Dynamics of serrated flow in a bulk metallic glass," AIP Advances, vol. 1, no. 3, p. 032158, 2011.
[2] D. Klaumünzer, A. Lazarev, R. Maaß, F. H. Dalla Torre, A. Vinogradov, and J. F. Löffler, "Probing Shear-Band Initiation in Metallic Glasses," Physical Review Letters, vol. 107, no. 18, 2011.

[3] M. L. Falk and J. S. Langer, "Dynamics of viscoplastic deformation in amorphous solids," Physical Review E: Statistical Physics, Plasmas, Fluids, and Related Interdisciplinary Topics, vol. 57, no. 6, pp. 7192-7205, 1998.

[4] Y. Q. Cheng, Z. Han, Y. Li, and E. Ma, "Erratum: Cold versus hot shear banding in bulk metallic glass," Physical Review B: Condensed Matter and Materials Physics, vol. 80, no. 22, 2009.

[5] B. A. Sun, H. B. Yu, W. Jiao, H. Y. Bai, D. Q. Zhao, and W. H. Wang, "Plasticity of Ductile Metallic Glasses: A Self-Organized Critical State," Physical Review Letters, vol. 105, no. 3, 2010.

[6] R. Dasgupta, H. G. Hentschel, and I. Procaccia, "Microscopic Mechanism of Shear Bands in Amorphous Solids," Physical Review Letters, vol. 109, no. 25, 2012.

[7] X. Guo, X. Xie, J. Ren et al., "Plastic dynamics of the $\mathrm{Al}_{0.5} \mathrm{CoCrCuFeNi}$ high entropy alloy at cryogenic temperatures: Jerky flow, stair-like fluctuation, scaling behavior, and nonchaotic state," Applied Physics Letters, vol. 111, no. 25, 2017.

[8] J. L. Ren, C. Chen, Z. Y. Liu, R. Li, and G. Wang, "Plastic dynamics transition between chaotic and self-organized critical states in a glassy metal via a multifractal intermediate," Physical Review B: Condensed Matter and Materials Physics, vol. 86, no. 13, 2012.

[9] J. Ren, C. Chen, G. Wang, and P. K. Liaw, "Transition of Temporal Scaling Behavior in Percolation Assisted Shear-branching Structure during Plastic Deformation," Scientific Reports, vol. 7, no. 1, 2017.

[10] D. X. Han, G. Wang, J. L. Ren, L. P. Yu et al., "Stick-slip dynamics in a $\mathrm{Ni} 62 \mathrm{Nb} 38$, metallic glass film during nanoscratching," Acta Materialia, vol. 136, pp. 49-60, 2017.

[11] S. V. Ketov, Y. H. Sun, S. Nachum et al., "Rejuvenation of metallic glasses by non-affine thermal strain,” Nature, vol. 524, no. 7564, pp. 200-203, 2015.

[12] K.-S. Yoon, M. Lee, E. Fleury, and J.-C. Lee, "Cryogenic temperature plasticity of a bulk amorphous alloy," Acta Materialia, vol. 58, no. 16, pp. 5295-5304, 2010.

[13] Y. Huang, W. Zheng, F. He, and J. Shen, "The temperature dependent dynamic mechanical response of a $\mathrm{ZrCuNiAl}$ bulk metallic glass," Materials Science and Engineering: A Structural Materials: Properties, Microstructure and Processing, vol. 551, pp. 100-103, 2012.

[14] C. Liu, V. Roddatis, P. Kenesei, and R. Maaß, "Shear-band thickness and shear-band cavities in a Zr-based metallic glass," Acta Materialia, vol. 140, pp. 206-216, 2017.

[15] Z. Lu, W. Jiao, W. Wang, and H. Bai, "Flow Unit Perspective on Room Temperature Homogeneous Plastic Deformation in Metallic Glasses," Physical Review Letters, vol. 113, no. 4, 2014.

[16] W. L. Johnson and K. Samwer, “A Universal Criterion for Plastic Yielding of Metallic Glasses with a ," Physical Review Letters, vol. 95, no. 19, 2005.

[17] A. Zaheri, F. Abdeljawad, and M. Haataja, "Simulation study of mechanical properties of bulk metallic glass systems: Martensitic inclusions and twinned precipitates," Modelling and Simulation in Materials Science and Engineering, vol. 22, no. 8, 2014.

[18] J. Ren, C. Chen, G. Wang et al., "Various sizes of sliding event bursts in the plastic flow of metallic glasses based on a spatiotemporal dynamic model," Journal of Applied Physics, vol. 116, no. 3, p. 033520, 2014. 
[19] Z. Han, W. F. Wu, Y. Li, Y. J. Wei, and H. J. Gao, "An instability index of shear band for plasticity in metallic glasses," Acta Materialia, vol. 57, no. 5, pp. 1367-1372, 2009.

[20] D. Bigoni and F. Dal Corso, "The unrestrainable growth of a shear band in a prestressed material," Proceedings of the Royal Society A Mathematical, Physical and Engineering Sciences, vol. 464, no. 2097, pp. 2365-2390, 2008.

[21] V. A. Levashov, J. R. Morris, and T. Egami, "Viscosity, Shear Waves, and Atomic-Level Stress-Stress Correlations," Physical Review Letters, vol. 106, pp. 162-172, 2001.

[22] S. Wiggins, Introduction to Applied Nonlinear Dynamical Systems and Chaos, vol. 2, Springer, New York, NY, USA, 2003.

[23] Q. Wang, C. Lu, G. G. Ye, and L. H. Dai, "Modelling the tuned criticality in stick-slip friction during metal cutting," Modelling and Simulation in Materials Science and Engineering, vol. 23, no. 5, p. 055013, 2015.

[24] G. Wang, Y. N. Han, X. H. Xu, F. J. Ke, B. S. Han, and W. H. Wang, "Ductile to brittle transition in dynamic fracture of brittle bulk metallic glass," Journal of Applied Physics, vol. 103, no. 9, p. 093520, 2008.

[25] S. M. Pincus, "Approximate entropy as a measure of system complexity," Proceedings of the National Acadamy of Sciences of the United States of America, vol. 88, no. 6, pp. 2297-2301, 1991.

[26] A. H. Nayfeh and D. T. Mook, Nonlinear Oscillations, John Wiley \& Sons, New York, NY, USA, 1979.

[27] C. Chen, J. Ren, G. Wang, K. A. Dahmen, and P. K. Liaw, "Scaling behavior and complexity of plastic deformation for a bulk metallic glass at cryogenic temperatures," Physical Review E: Statistical, Nonlinear, and Soft Matter Physics, vol. 92, no. 1, 2015.

[28] J. Huang and D. L. Turcotte, "Are earthquakes an example of deterministic chaos?" Geophysical Research Letters, vol. 17, no. 3, pp. 223-226, 1990.

[29] F. B. Pelap, L. Y. Kagho, and C. F. Fogang, "Chaotic behavior of earthquakes induced by a nonlinear magma up flow," Chaos, Solitons \& Fractals, vol. 87, pp. 71-83, 2016.

[30] Y. Iwashita and I. Nakanishi, "Scaling laws of earthquakes derived by renormalization group method," Chaos, Solitons \& Fractals, vol. 24, no. 2, pp. 511-518, 2005.

[31] P. Bak and C. Tang, "Earthquakes as a self-organized critical phenomenon," Journal of Geophysical Research: Solid Earth, vol. 94, no. B11, pp. 15635-15637, 1989.

[32] J. D. Phillips, "Instability and chaos in hillslope evolution," American Journal of Science, vol. 293, no. 1, pp. 25-48, 1993.

[33] M. Bernardo, P. Kowalczyk, and A. Nordmark, "Sliding bifurcations: a novel mechanism for the sudden onset of chaos in dry friction oscillators," International Journal of Bifurcation and Chaos, vol. 13, no. 10, pp. 2935-2948, 2003.

[34] Q. Sun, "Nonlinear Motion Behavior Or Rock Mass under Quasi-Periodic Driving," Metal Mine, vol. 393, pp. 35-38, 2009.

[35] R. Burridge and L. Knopoff, "Model And Theoretical Seismicity," Bulletin of the Seismological Society of America, vol. 57, pp. 341-371, 1967.

[36] B. A. Sun and W. H. Wang, "Fractal nature of multiple shear bands in severely deformed metallic glass," Applied Physics Letters, vol. 98, no. 20, Article ID 201902, 2011. 


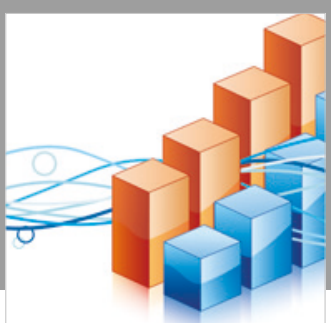

Advances in

Operations Research

\section{-n-m}
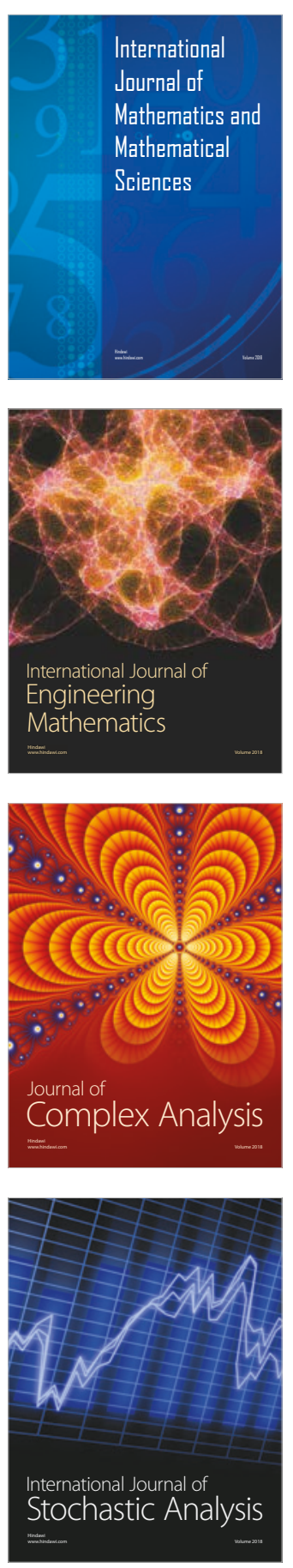
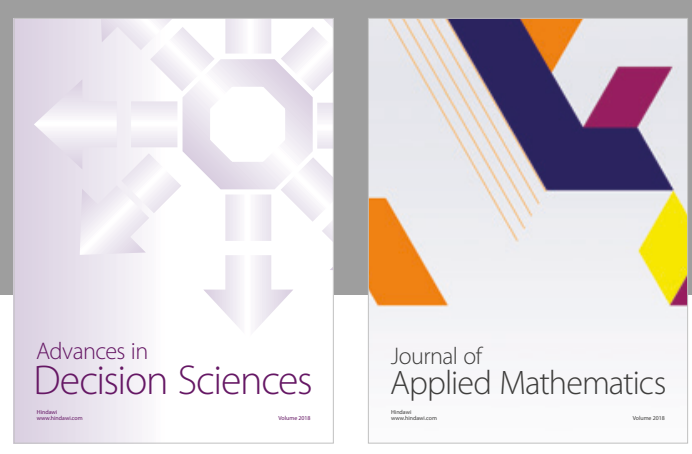

Journal of

Applied Mathematics
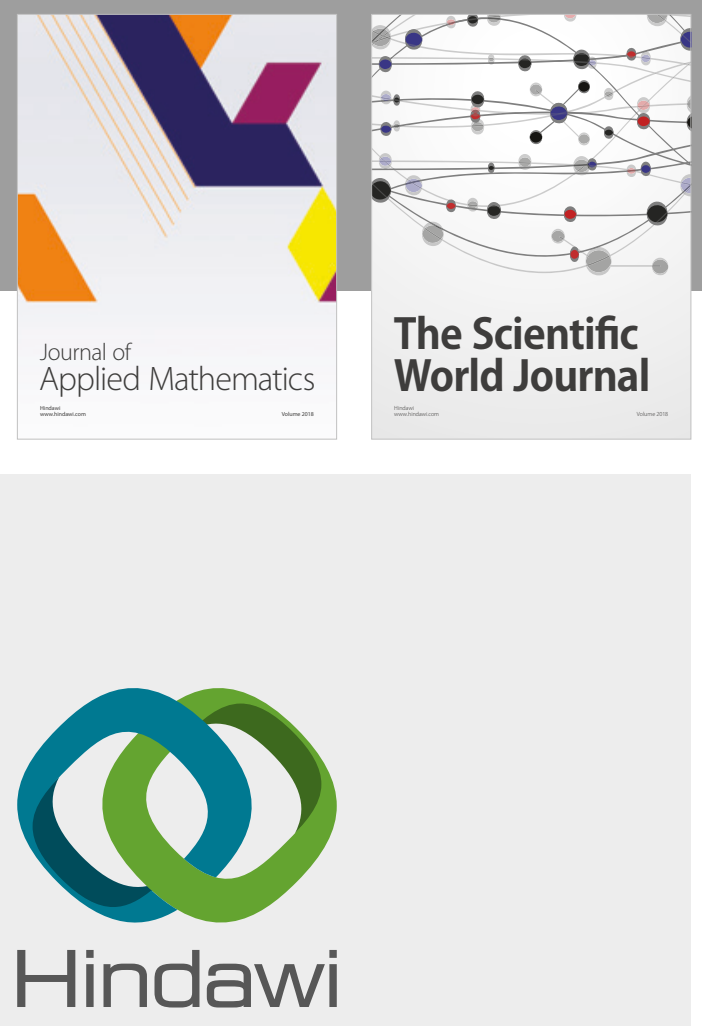

Submit your manuscripts at

www.hindawi.com

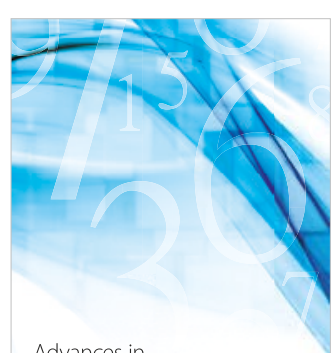

Advances in
Numerical Analysis
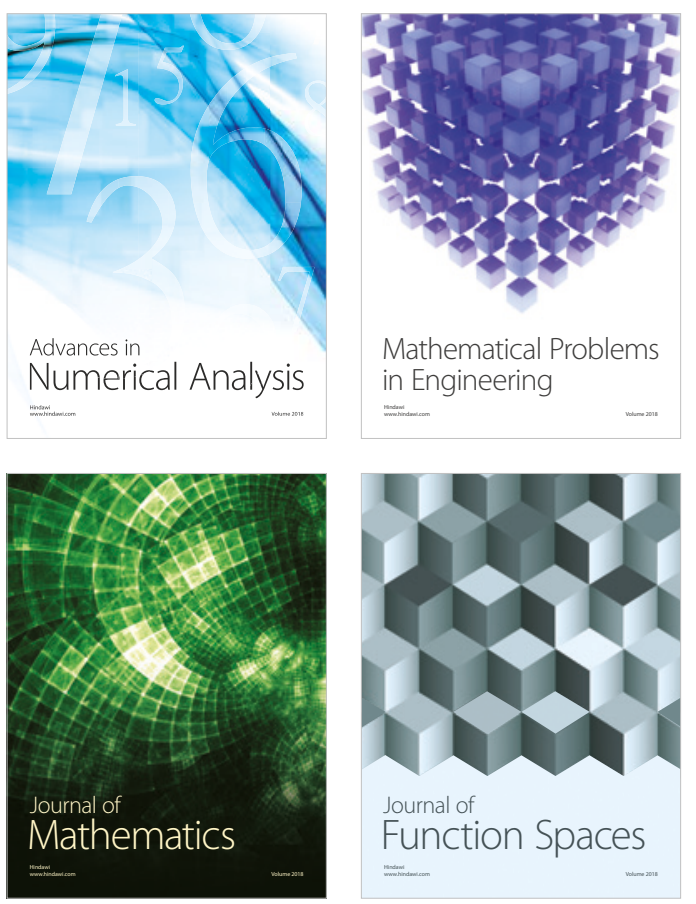

Mathematical Problems in Engineering

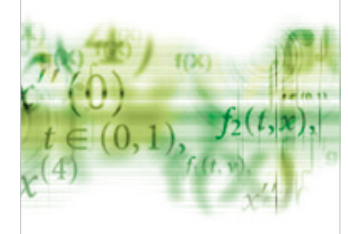

International Journal of

Differential Equations

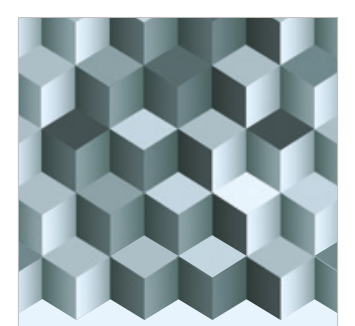

Journal of

Function Spaces
The Scientific

World Journal

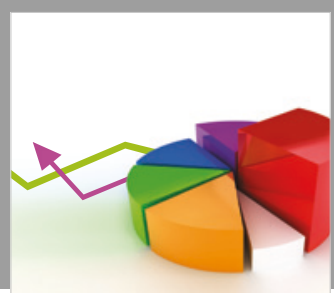

Journal of

Probability and Statistics
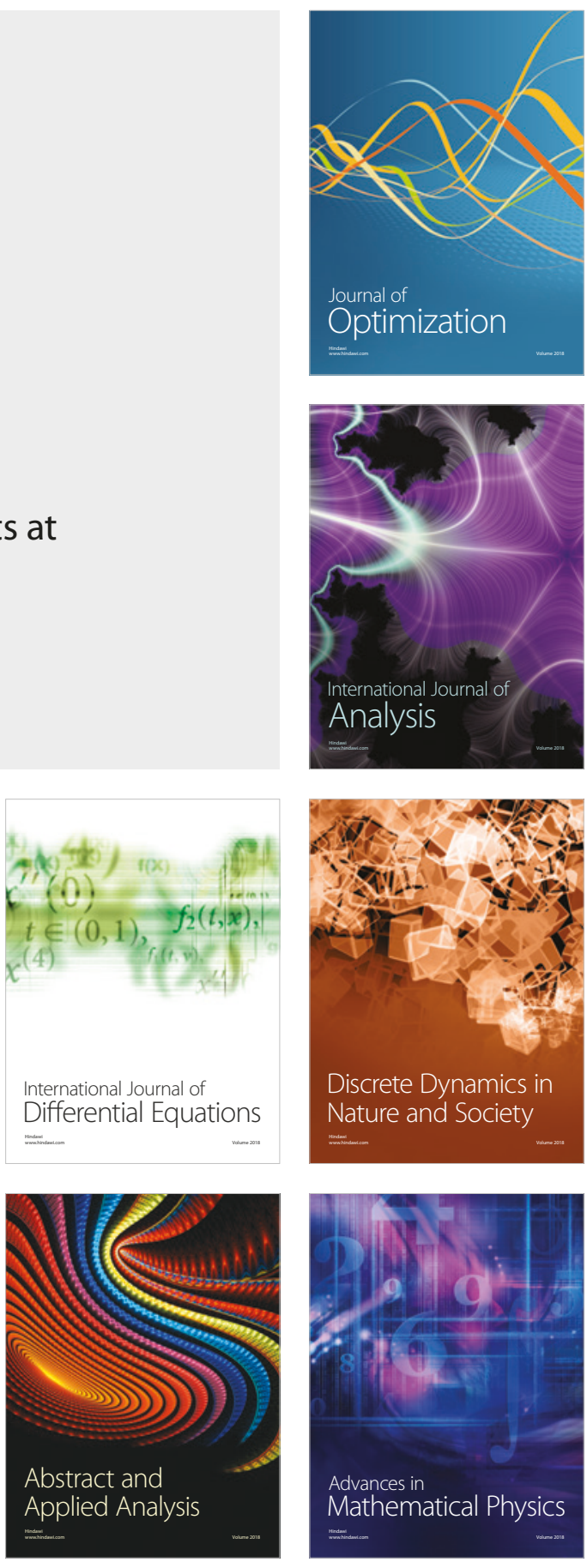